\title{
Adaptive Extreme Load Estimation in Wind Turbines
}

\author{
Qiyun Pan* and Eunshin Byon* \\ University of Michigan, Ann Arbor, Michigan 48109, United States
}

\begin{abstract}
To help wind turbine reliability analysis in a design stage, aeroelastic simulators have been developed to generate stochastic load responses imposed on a wind turbine structure, given pre-specified turbulent wind conditions. In particular, system designers can use the simulators to estimate the extreme load responses during a turbine's design life. However, it has been shown that using the crude Monte Carlo sampling methods to simulate the extreme load associated with a small load exceeding probability is computationally prohibitive, and the estimation results are highly uncertain. Importance sampling methods can overcome the limitations of the crude Monte Carlo sampling methods. We develop adaptive algorithms to iteratively refine the importance sampling density to efficiently estimate the extreme load.
\end{abstract}

\section{Nomenclature}

$P_{T} \quad$ Target probability of load exceedance

$l_{T} \quad$ Extreme load

$X \quad$ Input vector

$Y \quad$ Output variable

$p_{X} \quad$ Original input density of $\mathrm{X}$

$q_{X} \quad$ Importance sampling density

$\theta \quad$ Parameter in the importance sampling density

\section{Introduction}

To achieve reliable operations during a wind turbine's design life, it is crucial to quantitatively estimate the expected loads imposed on turbine subsystems over a range of dynamic operating conditions. ${ }^{1}$ Recent technology advancements provide opportunities to quantify loads and their variability in the design stage. For example, the U.S Department of Energy's National Renewable Energy Laboratory (NREL) has developed aeroelastic simulators to help design reliable turbines. ${ }^{2,3}$ The system designer specifies the turbine design parameters, and feeds a random weather condition as input into the simulators. Then, the simulators generate structural and mechanical load responses at turbine subsystems. In particular, the NREL simulators use a stochastic computer model which generates a random output even when the input is fixed. This is because the NREL simulators embed more than 8 million random variables, so that the output (e.g., blade tip deflection) is not uniquely determined even at the same simulation input (i.e., weather condition). ${ }^{2}$ In this study, we call such simulators as "stochastic simulation models". On the contrary, some simulators use deterministic computer models that generate deterministic output at a fixed input. We refer to those simulators as "deterministic simulation models" in this study.

In the reliability-based wind turbine design, the International Electrotechnical Commission (IEC) design standard's design load case (DLC) 1.1 requires assessing the extreme loads during a turbine's normal operations. ${ }^{4}$ Specifically, a turbine designer should estimate the turbine's extreme load level $l_{T}$, given the target

\footnotetext{
${ }^{*}$ The Department of Industrial and Operations Engineering, 1891 IOE Building, 1205 Beal Avenue, Ann Arbor, MI 481092117
} 
probability of load exceedance (POE) $P_{T}$, or load exceedance probability, defined as

$$
\begin{aligned}
P_{T} & =P\left(Y>l_{T}\right) \\
& =\int_{X} P\left(Y>l_{T} \mid X=x\right) \cdot p_{X}(x) d x,
\end{aligned}
$$

where $p_{X}$ is the probability density function of the environmental random vector $X$, and $Y$ is the simulation output describing the load response of interest.

Typically, 10-minute turbine operations are simulated for each run, which can be used to evaluate a target $P_{T}$ corresponding to a turbine's design life (or return period) of $T$ years as follows. ${ }^{5}$

$$
\begin{aligned}
P_{T} & =\frac{10 \text { minutes }}{T \text { years } \times 365.25 \text { days } \times 24 \text { hours } \times 60 \text { minutes }} \\
& \approx \frac{1.9 \times 10^{-5}}{T} .
\end{aligned}
$$

For example, when a turbine's design life is 50 years, the target POE $P_{T}$ becomes approximately $3.8 \times 10^{-7}$.

Given $P_{T}$, the extreme load $l_{T}$ during $T$-year design life is defined as

$$
l_{T}=\inf \left\{\theta \in \mathbb{R}: P(Y>\theta) \leq P_{T}\right\},
$$

where "inf" represents the infimum. ${ }^{6}$ Mathematically, the extreme load $l_{T}$ relates to the extreme quantile associated with the right tail probability $P_{T}$ in the distribution of $Y$ (Figure 1).

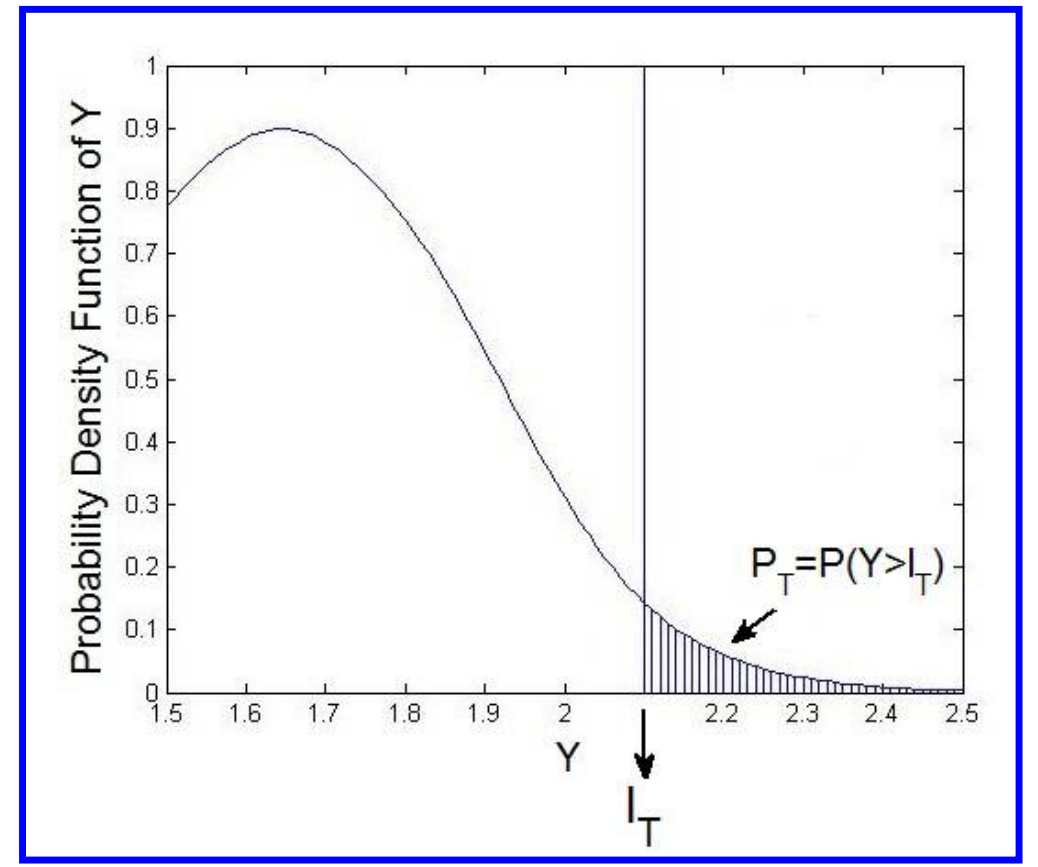

Figure 1: Illustration of extreme load $l_{T}$ corresponding to the target POE $P_{T}$

When $P_{T}$ is small, estimating the extreme quantile faces large estimation uncertainties and computational difficulties. The objective of this study is to develop a new method that enables computationally efficient extreme load assessments of wind turbines through effective use of stochastic simulation models.

\section{Problem Background}

The crude Monte Carlo (CMC) sampling is the default practice to run the stochastic simulator, namely, sample the input $x^{\prime}$ s from its probability density function $p_{X}(x)$ and use the sampled $x^{\prime}$ s to run the simulator and produce $y^{\prime}$ s until a sufficient number of output samples are obtained. Let $y_{j}$ denote the $j^{\text {th }}$ output of 
response $Y$ and $N_{T}$ denote the total number of simulation runs. Using the CMC, the POE estimator at level $l_{T}$ is

$$
\hat{P}_{C M C}\left(Y>l_{T}\right)=\frac{1}{N_{T}} \sum_{j=1}^{N_{T}} \mathbb{1}\left(y_{j}>l_{T}\right),
$$

and the extreme load estimator corresponding to the target POE level $P_{T}$ is given by

$$
\hat{l}_{T, C M C}=\inf \left\{\theta \in \mathbb{R}: \hat{P}_{C M C}(Y>\theta) \leq P_{T}\right\} .
$$

With small $P_{T}$, to estimate $\hat{l}_{T, C M C}$, CMC requires enormous computational efforts. For example, to estimate extreme loads for $50 \sim 100$ years, Manuel et al. ${ }^{7}$ use a Linux computer cluster with 1,024 cores. Moreover, the estimation results from CMC are highly uncertain, leading to large estimation variance. ${ }^{6}$

To overcome the limitations of CMC, importance sampling methods have been used to improve the simulation efficiency and reduce the estimation uncertainty. The underlying idea of the importance sampling methods is to generate random input samples from a biased distribution rather than the original input density $p_{X}(x)$, so that the input regions that generate large $y$ 's can get more sampling efforts. As a result, the estimation efficiency with importance sampling can be significantly improved compared with that of CMC. Due to its powerful performance, the importance sampling methods have been widely used in the reliability analysis, ${ }^{8}$ but most studies have been limited to the deterministic simulation models.

Recently Choe et al. ${ }^{9}$ introduce the importance sampling for the POE estimation with stochastic simulation models, called a stochastic importance sampling method. In Choe et al., ${ }^{9}$ two important sampling methods are introduced. The first method allows multiple simulation runs at each sampled input to account for the randomness in the simulation output, whereas the second method runs the simulation once at each input. It has been shown that the estimation performance of the two methods is similar. Because the second method is a special case of the first method, we summarize the first method in our subsequent discussions.

Let $x_{i}, i=1,2, \ldots, M$, denote the samples of input vector from the importance sampling density $q_{X}(x)$, and $M$ be the input sample size. At each $x_{i}$, we run simulations $N_{i}$ times to obtain $N_{i}$ outputs of $y_{i j}$, $j=1,2, \ldots, N_{i}$. The POE estimator of the probability that $Y$ exceeds level $l_{T}$, denoted as $\hat{P}_{I S}\left(Y>l_{T}\right)$, is

$$
\hat{P}_{I S}\left(Y>l_{T}\right)=\frac{1}{M} \sum_{i=1}^{M}\left(\frac{1}{N_{i}} \sum_{j=1}^{N_{i}} \mathbb{1}\left(y_{i j}>l_{T}\right)\right) \frac{p_{X}\left(x_{i}\right)}{q_{X}\left(x_{i} ; l_{T}\right)} .
$$

Choe et al. ${ }^{9}$ show that the optimal importance sampling density that minimizes the variance of $\hat{P}_{I S}(Y>$ $\left.l_{T}\right)$, is given by

$$
q_{X}\left(x ; l_{T}\right)=\frac{1}{C_{q}} p_{X}(x) \sqrt{\frac{1}{N_{T}} s\left(x ; l_{T}\right)\left(1-s\left(x ; l_{T}\right)\right)+s\left(x ; l_{T}\right)^{2}},
$$

where $C_{q}=\int p_{X}(x) \sqrt{\frac{1}{N_{T}} s\left(x ; l_{T}\right)\left(1-s\left(x ; l_{T}\right)\right)+s\left(x ; l_{T}\right)^{2}}$ is the normalizing constant. Moreover, the optimal allocation $N_{i}$ at each $x_{i}, i=1, \ldots, M$, that collaboratively minimizes the variance of $\hat{P}_{I S}\left(Y>l_{T}\right)$ is given by:

$$
N_{i}=N_{T} \frac{\sqrt{\frac{N_{T}\left(1-s\left(x_{i} ; l_{T}\right)\right)}{1+\left(N_{T}-1\right) s\left(x_{i} ; l_{T}\right)}}}{\sum_{j=1}^{M} \sqrt{\frac{N_{T}\left(1-s\left(x_{i} ; l_{T}\right)\right)}{1+\left(N_{T}-1\right) s\left(x_{i} ; l_{T}\right)}}}, i=1, \ldots, M,
$$

where $N_{T}$ is the number of total simulation runs. Here, $s\left(x ; l_{T}\right)$ represents the conditional probability that the response $Y$ exceeds $l_{T}$, given a realization $x$ of the input $X$, i.e.,

$$
s\left(x ; l_{T}\right)=P\left(Y>l_{T} \mid X=x\right) .
$$

Correspondingly, the importance sampling estimator for the extreme quantile $l_{T}$ is given by ${ }^{6}$

$$
\hat{l}_{T, I S}=\inf \left\{\theta \in \mathbb{R}: \hat{P}_{I S}(Y>\theta) \leq P_{T}\right\} .
$$


Choe et al. ${ }^{6}$ integrate the importance sampling method with the order statistics theories to improve the extreme load estimation accuracy, given the computational budget $N_{T}$. Suppose $y_{(j)}, j=1, \cdots, N_{T}$, denote the $j^{\text {th }}$ smallest order statistic of the output samples. In Equation (8), by replacing $l_{T}$ with $y_{(j)}, \hat{P}\left(Y>y_{(j)}\right)$ can be obtained in ascending order at all $y_{(j)}$, where $j$ goes from $N_{T}$ to 1 as shown in Figure 2 (Note that the POE estimate at the largest sample $y_{\left(N_{T}\right)}$ is 0 from Equation (8), so the smallest nonzero POE estimate starts from $\left.y_{\left(N_{T}-1\right)}\right)$. From Equation $(12), y_{(j)}$ can be interpreted as the extreme quantile estimate at POE level $\hat{P}\left(Y>y_{(j)}\right)$ for each $j=1, \cdots, N_{T}$. As a result, for a small $P_{T} \in[0,1]$, we can get the extreme load estimate corresponding to each order statistic among the $N_{T}$ output samples. Detailed procedure for estimating the extreme load with the importance sampling method is provided by Choe et al. ${ }^{6}$

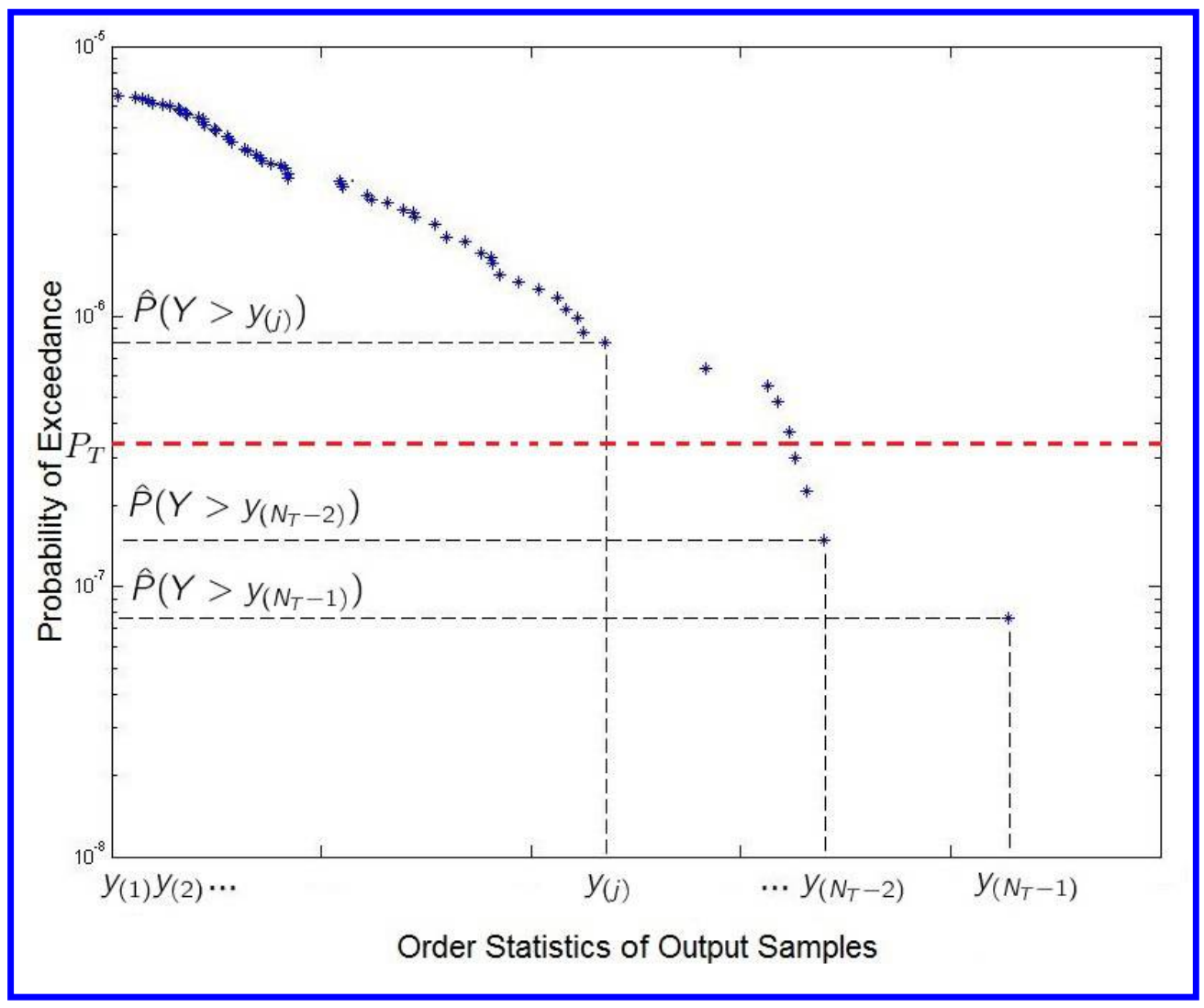

Figure 2: Extreme load estimates with order statistics

These studies by Choe et al. ${ }^{6,9}$ show the effectiveness of importance sampling theories in estimating the wind turbine reliability and extreme load estimations. However, one notable limitation is that to achieve the optimal estimation of $l_{T}$ using (12), the parameter $\theta$ in the IS density $q_{X}(x ; \theta)$ should be the target extreme load $l_{T}$, but $l_{T}$ is the value we want to estimate, which is unknown.

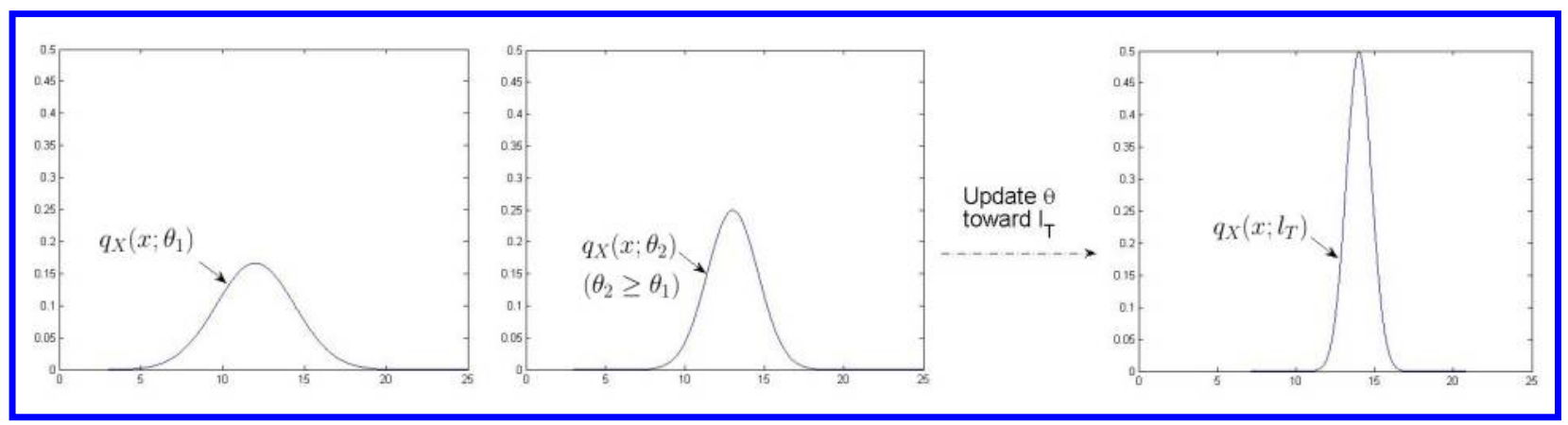

Figure 3: Proposed idea: iterative updating of parameter $\theta$ in the IS density 
Faced with this problem, we develop adaptive sampling method to iteratively choose the proper parameter $\theta$. Our idea is to start with a small parameter $\theta$ and increase $\theta$ toward $l_{T}$ as large output samples are obtained, as shown in Figure 3.

\section{Adaptive Importance Sampling for Extreme Load Estimation}

To develop the adaptive sampling method for the extreme load estimation, we borrow the idea from Morio, ${ }^{10}$ who presents a $\rho$-quantile method to estimate the extreme quantile in the deterministic simulation model. The basic idea is to update the parameter $\theta$ using the upper $\rho$ percent of output samples in each iteration. Given the limited computational resource, we proceed the iteration $K$ times. Suppose that we start with a relatively small $\theta_{1}$ in $q_{X}(x ; \theta)$ and run simulation $n$ times to get $n$ outputs, $y_{1}, \ldots, y_{n}$. These samples will be generally greater than $\theta_{1}$. Then, we update $\theta_{1}$ to a greater level $\theta_{2}$ by selecting one of the output samples $y_{1}, \ldots, y_{n}$. Specifically, we use the $\lceil n(1-\rho)\rceil^{\text {th }}$ smallest sample from the outputs, where $\lceil a\rceil$ is the smallest integer greater than, or equal to, $a$. Let $y_{(i)}(i=1, \cdots, n)$ denote the $i^{\text {th }}$ smallest ordered statistics. Then, we update the parameter $\theta_{1}$ to $\theta_{2}=y_{(\lceil n(1-\rho)\rceil)}$ in the next iteration. However, in the updating process, we need to guarantee $\theta$ being no greater than the target extreme load $l_{T}$ to have unbiased POE estimates. Under this restriction, we apply two different updating mechanisms.

First, we start with a safe parameter $\theta=\theta_{1}$. In the beginning when we use a small $\theta$ in the IS density $q_{X}(x ; \theta)$, the output samples tend to be greater than $\theta$, but are not likely to be large enough to estimate the small target POE. Let $Y_{\text {sec-max }}^{1: k}$ denote the second largest output sample we obtain up to the $k^{\text {th }}$ iteration, which provides the smallest POE estimate up to the $k^{t h}$ iteration. When the current smallest POE estimate with $Y_{s e c-\text { max }}^{1: k}$ is greater than $P_{T}$, we cannot estimate the target extreme load $l_{T}$ associated with $P_{T}$ yet. To obtain larger outputs in the subsequent iterations, we update the parameter $\theta$ with the $\rho$-quantile of the output samples obtained in the current iteration.

Second, as the parameter $\theta$ goes up, we will obtain large output samples. After some iterations, the smallest POE estimate will reach $P_{T}$ and the extreme quantile can be estimated with the output samples obtained up to the current iteration. Then, care should be given to prevent the parameter $\theta$ from exceeding the potential extreme quantile $l_{T}$ in the next iteration. We define $W_{k}$, which is the largest output sample at which the corresponding estimated $\mathrm{POE}$ is greater than $P_{T}$, and update the parameter with the minimum of $\rho$-quantile and $W_{k}$.

We summarize the proposed adaptive sampling algorithm as follows. Let $N$ denote the number of output samples we obtain in each iteration.

Step 0 . Set $k=1$ and a low value for $\theta_{1}$. Set $\rho \in(0,1)$ such that $\rho N \geq 2$.

Step 1. Obtain $q_{X}\left(x ; \theta_{k}\right)$ with parameter $\theta_{k}$ in the $k^{\text {th }}$ iteration.

Step 2. Sample $x_{i}^{k}, i=1, \ldots, M$, from $q_{X}\left(x ; \theta_{k}\right)$.

Step 3. Determine the allocation size $N_{i}^{k}$ for each $x_{i}^{k}, i=1, \ldots, M$, using Equation (10).

Step 4. Run simulation $N_{i}^{k}$ times at each $x_{i}^{k}$ to obtain $N_{i}^{k}$ outputs, $y_{i j}^{k}\left(j=1, \cdots, N_{i}^{k}\right)$ for each $i(i=$ $1, \cdots, M)$. Let $y_{(t)}^{1: k}$ denote the $t^{t h}$ order statistic of outputs that satisfies $y_{(t)}^{1: k} \geq \max _{1 \leq s \leq k}\left\{\theta_{s}\right\}$ among all samples from 1 to the $k^{t h}$ iteration, and $N_{e x c}^{1: k}$ be the total number of output samples exceeding $\max _{1 \leq s \leq k}\left\{\theta_{s}\right\}$ up to the $k^{t h}$ iteration. For each $y_{(t)}^{1: k x} \geq \max _{1 \leq s \leq k}\left\{\theta_{s}\right\}, t=1, \ldots, N_{e x c}^{1: k}$, use all $k N_{T}$ samples of $y_{i j}^{s}, s=1, \ldots, k$ to compute

$$
\hat{P}_{k}\left(y_{(t)}^{1: k}\right)=\frac{1}{k} \sum_{s=1}^{k}\left(\frac{1}{M} \sum_{i=1}^{M}\left(\frac{1}{N_{i}^{s}} \sum_{j=1}^{N_{i}^{s}} \mathbb{1}\left(y_{i j}^{s}>y_{(t)}^{1: k}\right)\right) \frac{p_{X}\left(x_{i}^{s}\right)}{q_{X}\left(x_{i}^{s} ; \theta_{s}\right)}\right) .
$$

Step 5 . If $k=K$, go to step 6 . Otherwise, update the parameter $\theta$ as follows.

A. If $\min _{t=1, \ldots, N_{e x c}^{1: k}}\left\{\hat{P}_{k}\left(y_{(t)}^{1: k}\right): \hat{P}_{k}\left(y_{(t)}^{1: k}\right)>0\right\}>P_{T}$, update the parameter as

$$
\theta_{k+1}=\gamma_{\rho}^{k}
$$


where $\gamma_{\rho}^{k}$ is the upper $\rho$-quantile of output samples in the $k^{\text {th }}$ iteration. Set $k=k+1$ and go back to step 1 .

B. Otherwise, set

$$
\begin{aligned}
W_{k} & =\max _{t=1, \ldots, N_{e x c}^{1: k}}\left\{y_{(t)}^{1: k}: \hat{P}_{k}\left(y_{(t)}^{1: k}\right)>P_{T}\right\} \\
\theta_{k+1} & =\min \left\{W_{k}, \gamma_{\rho}^{k}\right\} .
\end{aligned}
$$

Set $k=k+1$ and go back to step 1 .

Step 6. If $\left\{y_{(t)}^{1: K}: 0<\hat{P}_{K}\left(y_{(t)}^{1: K}\right) \leq P_{T}\right\} \neq \phi$, the extreme load estimate is given by

$$
\hat{Q}_{P_{T}, K}=\min _{t=1, \ldots, N_{e x c}^{1: K}}\left\{y_{(t)}^{1: K}: 0<\hat{P}_{K}\left(y_{(t)}^{1: K}\right) \leq P_{T}\right\} .
$$

Otherwise, the extreme $P_{T}$-quantile cannot be estimated given the computation resources.

Under the condition $y_{(t)}^{1: k} \geq \max _{1 \leq s \leq k}\left\{\theta_{s}\right\}, \hat{P}_{k}\left(y_{(t)}^{1: k}\right)$ in Equation (13) is an unbiased estimator for $P\left(Y>y_{(t)}^{1: k}\right)$, for each $t=1, \ldots, N_{e x c}^{1: k}$. Based on the above discussions, the IS density with any $\theta_{k}$, for $1 \leq k<K$, can be applied to unbiasedly estimate the POE level of $P_{T}$.

In the proposed approach, the $\rho$-quantile updating scheme is introduced to increase parameter $\theta$ from a small starting value towards the extreme quantile $l_{T}$. This algorithm controls the proposed parameter with newly obtained output samples in each iteration. We note that the value of $\rho$ should be selected carefully. When $\rho$ is too large, close to 1 , the $\rho$-quantile of output samples is small and the parameter $\theta$ will not increase rapidly as needed. On the contrary, when $\rho$ is too small, the number of upper $\rho$ percent output samples is small, which could lead to large variations in the $\rho$-quantile. For example, consider $N_{T}=1,000$ and $\rho=0.002$. The $\rho$-quantile becomes the second largest output sample. In general, this specific single sample has large variation in the stochastic simulation model. As a result, the variance of $\rho$-quantile is unavoidably increased when $\rho$ is too small, which possibly adds uncertainty to the extreme quantile estimation.

\section{Wind Turbine Extreme Load Estimation}

In this section, we apply the proposed algorithm to the wind turbine extreme load estimation with the set of NREL simulators, TurbSim ${ }^{2}$ and FAST. ${ }^{3}$ The input variable $X$ is 10 -minute average wind speed, which is assumed to follow a truncated Rayleigh distribution on the interval $[3,25](\mathrm{m} / \mathrm{s})$. The cumulative distribution function of Rayleigh distribution is $F(x)=1-e^{-x^{2} / 2 \tau^{2}}$ with the scale parameter $\tau=\sqrt{2 / \pi} \cdot 10$. With the input average wind speed, TurbSim generates 10 minutes time series of a 3D wind profile, and passes them to FAST. FAST simulates the load response $Y$ at different wind turbine components.

In this case study, we investigate two types of load responses, namely, the flapwise bending moment and the in-plane tip deflection. To obtain the IS density $q_{X}(x ; \theta)$ in $(9)$, we need $s(x ; \theta)$ in $(11)$. We take a pilot sample and fit the non-homogeneous GEV distribution with the output sample data and approximate $s(x, \theta)$. Detailed procedure to estimate $s(x ; \theta)$ is available in Choe et al. ${ }^{9}$

In our adaptive algorithm, we use the starting parameters $\theta_{1}=2.33(\mathrm{~m})$ and $\theta_{1}=14,600(\mathrm{kNm})$ for the in-plane tip deflection and the flapwise bending moment, respectively. In the standard IS that does not update $\theta$, we use these fixed parameters in the IS density. We use $\rho=0.1$. We use $N=300$, $M=50$ to implement 10 iterations for the adaptive algorithm. To maintain the same computational effort in comparing the extreme load estimation performance of the adaptive and the standard IS, the standard IS uses $N_{T}=300 \times 10=3,000$ and $M=50 \times 10=500$. Each extreme load estimation trajectory in Figure 4 and 5 is obtained by linear interpolation on 3,000 pairs of the output samples and their associated POE estimates.

Figure 4 demonstrates the 16 extreme load estimation trajectories for the in-plane tip deflection. Intervals $A_{I}$ and $B_{I}$ show the ranges of extreme load estimation at $P_{T}=10^{-5}$ using the proposed approach and standard IS respectively. The result shows that $A_{I}$ and $B_{I}$ ranges $[2.632,2.768](m)$ and $[2.625,2.805](m)$, respectively. The narrower range of $A_{I}$ indicates that the proposed approach reduces the extreme load estimation uncertainty, compared with the standard IS. Moreover, the minimum POE estimate using the 
proposed approach reaches $1.5 \times 10^{-6}$, which is smaller than $7.7 \times 10^{-6}$ obtained using the standard IS, with the same computational effort.

Figure 5 demonstrates the 20 extreme load estimation trajectories for the flapwise bending moment. Intervals $A_{F}$ and $B_{F}$ show the ranges of extreme load estimation at $P_{T}=1.1^{-4}$ using the proposed approach and standard IS, respectively. $A_{F}$ and $B_{F}$ ranges $[15157,15530](k N m)$ and $[15114,15640](k N m)$, respectively. In addition, the minimum POE estimates using the proposed approach are around $6.3 \times 10^{-5}$ $\sim 9.5 \times 10^{-5}$, which are smaller than that obtained using the standard IS $\left(9.5 \times 10^{-5} \sim 1.1 \times 10^{-4}\right)$, with the same computation resources. These results suggest the proposed approach has higher extreme load estimation accuracy and computation efficiency compared with the standard IS method. Figure 6 shows the parameter in IS density is updated through iterations from the low starting level 14,600 to 15, 210 in the proposed approach for estimating the extreme load of flapwise bending moment.

To further estimate the extreme load for a longer design life, say, 50 years, we conduct experiments with a larger $N_{T}$. We collect output samples from multiple experiments and construct a combined extreme load estimation trajectory. ${ }^{6}$ Figure 7 shows the extreme load estimation trajectories for the in-plane tip deflection. The trajectory is obtained from 40,000 ( $N=1,000$ in each iteration, 10 iterations and 4 repetitions) output samples. The largest estimated extreme load is $3.05(\mathrm{~m})$, which is associated with the POE level of $5.6 \times 10^{-8}$ (339-year return period). In particular, the 50-year extreme load estimate, which is associated with POE $3.8 \times 10^{-7}$, is $2.99(\mathrm{~m})$. This estimation is similar to those presented in Choe et al. ${ }^{6}$ which were obtained with larger computational runs.

Figure 8 shows the combined extreme load estimation trajectory for the flapwise bending moment. From the trajectory obtained from 240,000 $(N=1,000$ in each iteration, 10 iterations and 24 repetitions) output samples, it shows that the 50-year extreme load estimate associated with POE $3.8 \times 10^{-7}$ is $15980(\mathrm{kNm})$. Both results for in-plane tip deflection and flapwise bending moment indicate that our proposed approach provides a computationally feasible solution for the extreme load estimation with limited computational resources.

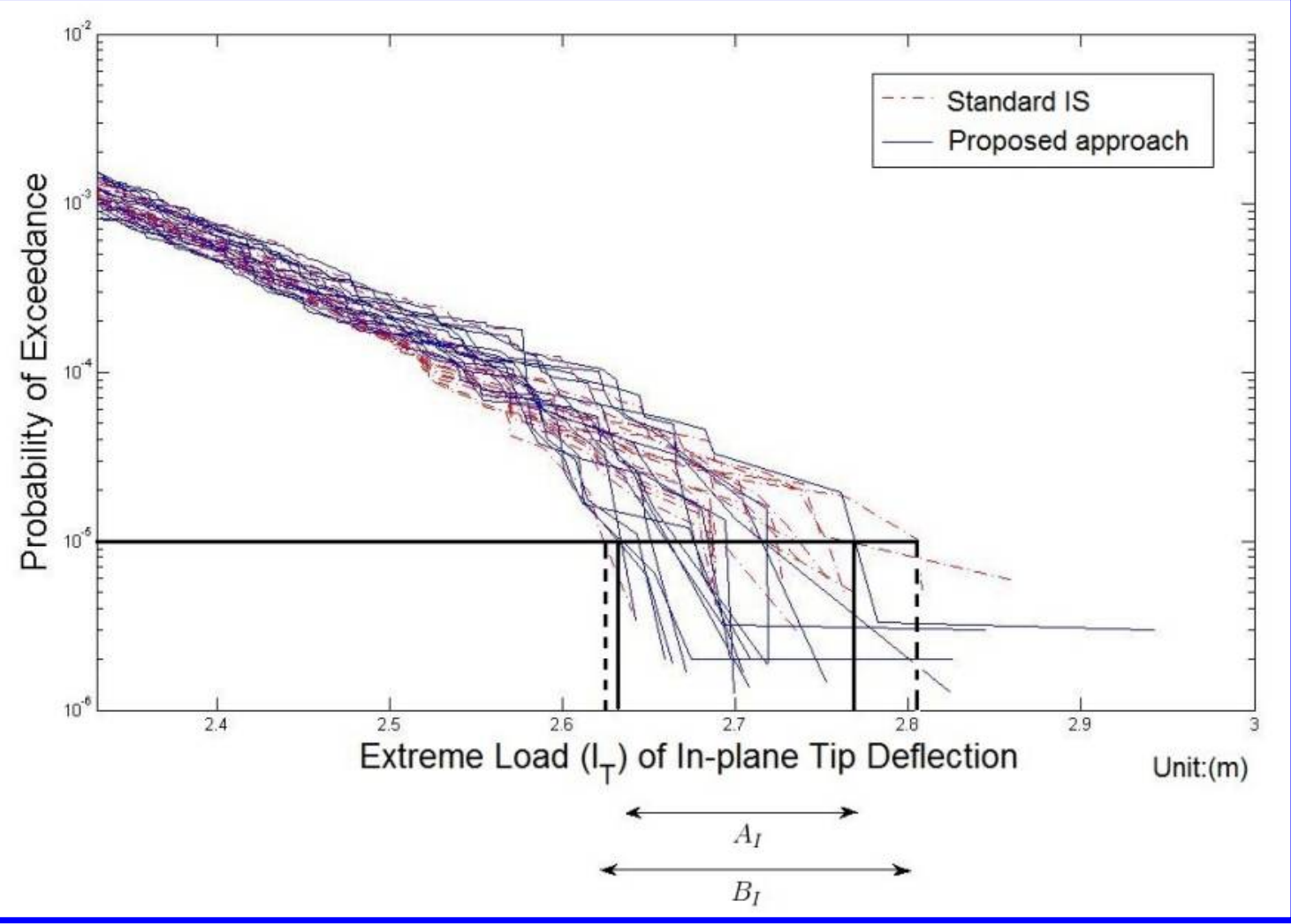

Figure 4: Extreme load estimation of in-plane tip deflection (For $P_{T}=10^{-5}$, the extreme load estimate from the proposed approach ranges from $2.63(\mathrm{~m})$ to $2.768(\mathrm{~m})$, indicated by interval $A_{I}$, whereas the extreme load estimate from the standard IS ranges from $2.625(\mathrm{~m})$ to $2.805(\mathrm{~m})$, indicated by interval $B_{I}$.) 


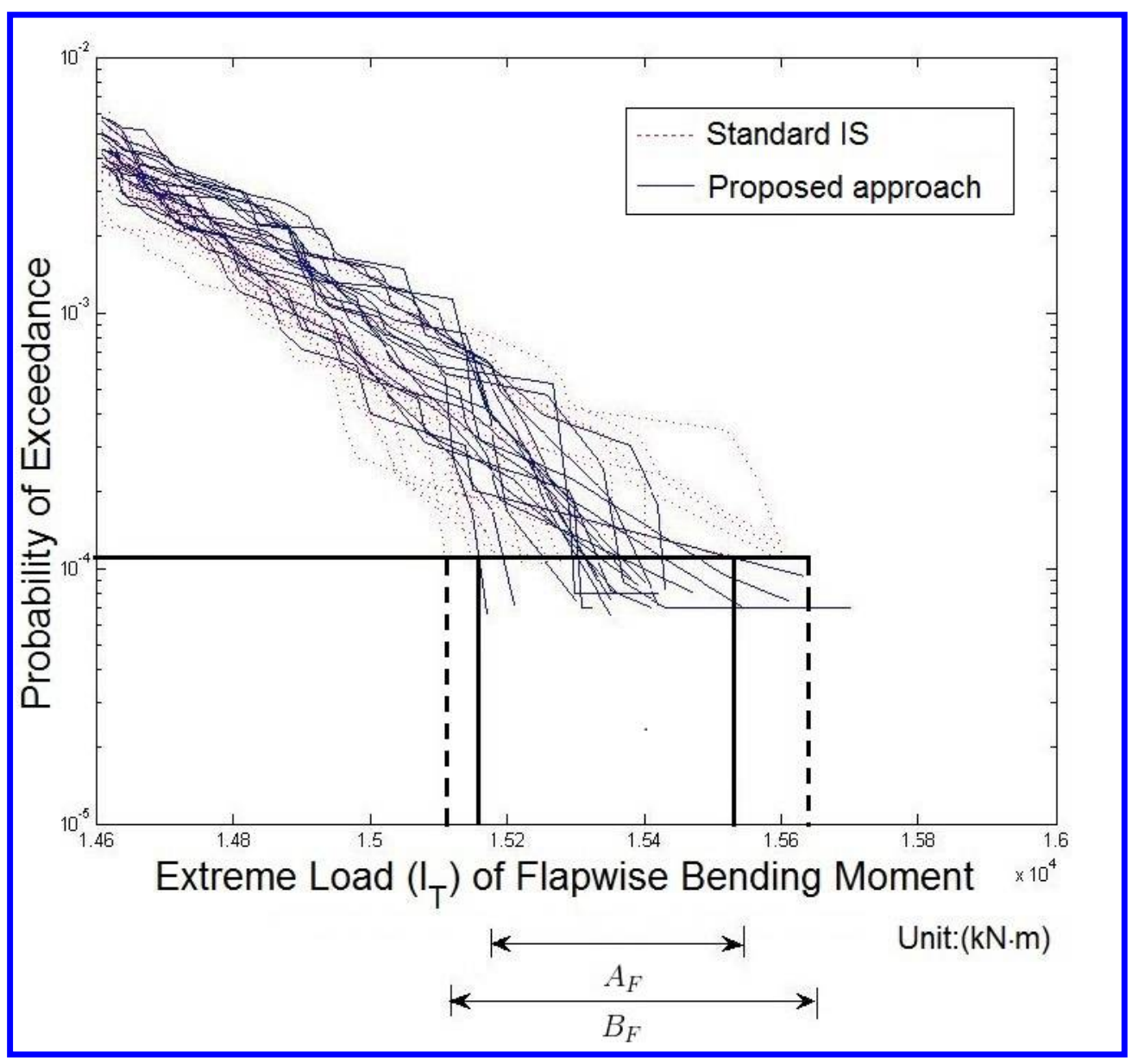

Figure 5: Extreme load estimation of flapwise bending moment (For $P_{T}=1.1 \times 10^{-4}$, the extreme load estimate from the proposed approach ranges from 15, $157(\mathrm{kNm})$ to $15,530(\mathrm{kNm})$, indicated by interval $A_{F}$, whereas the extreme load estimate from the standard IS ranges from $15,114(\mathrm{kNm})$ to $15,640(\mathrm{kNm})$, indicated by interval $B_{F}$.)

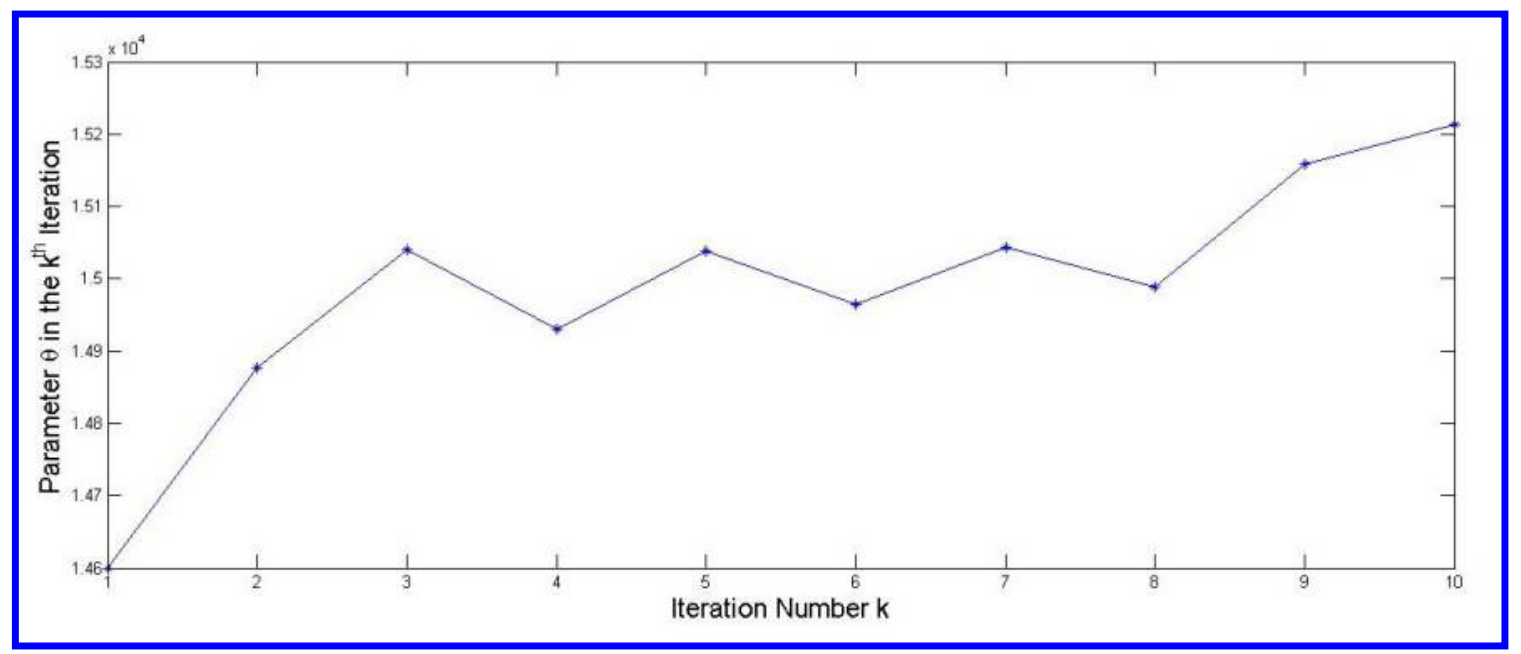

Figure 6: Updates of parameter $\theta$ in the IS density for estimating the extreme load of flapwise bending moment 


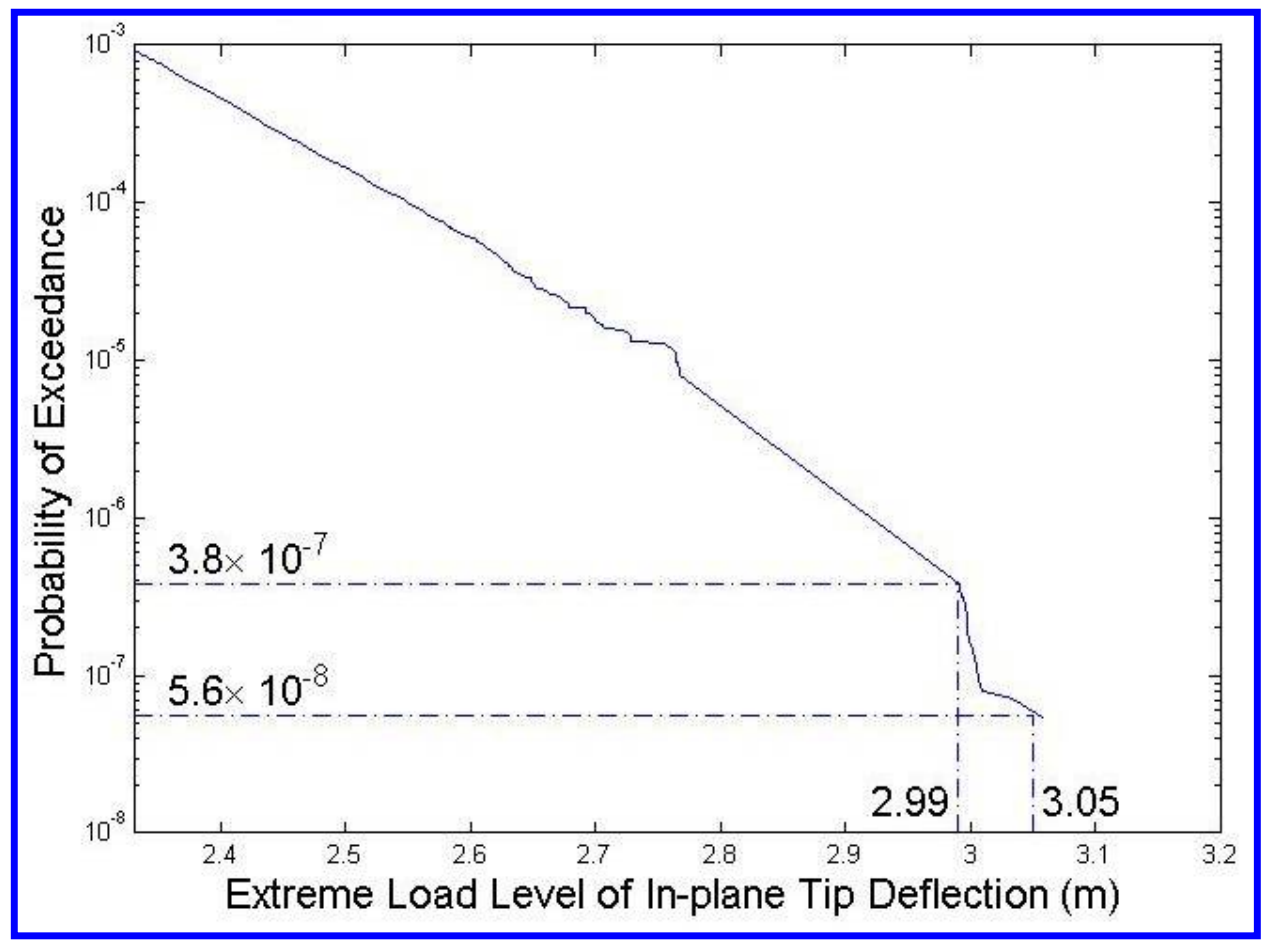

Figure 7: In-plane tip deflection extreme load estimation trajectory

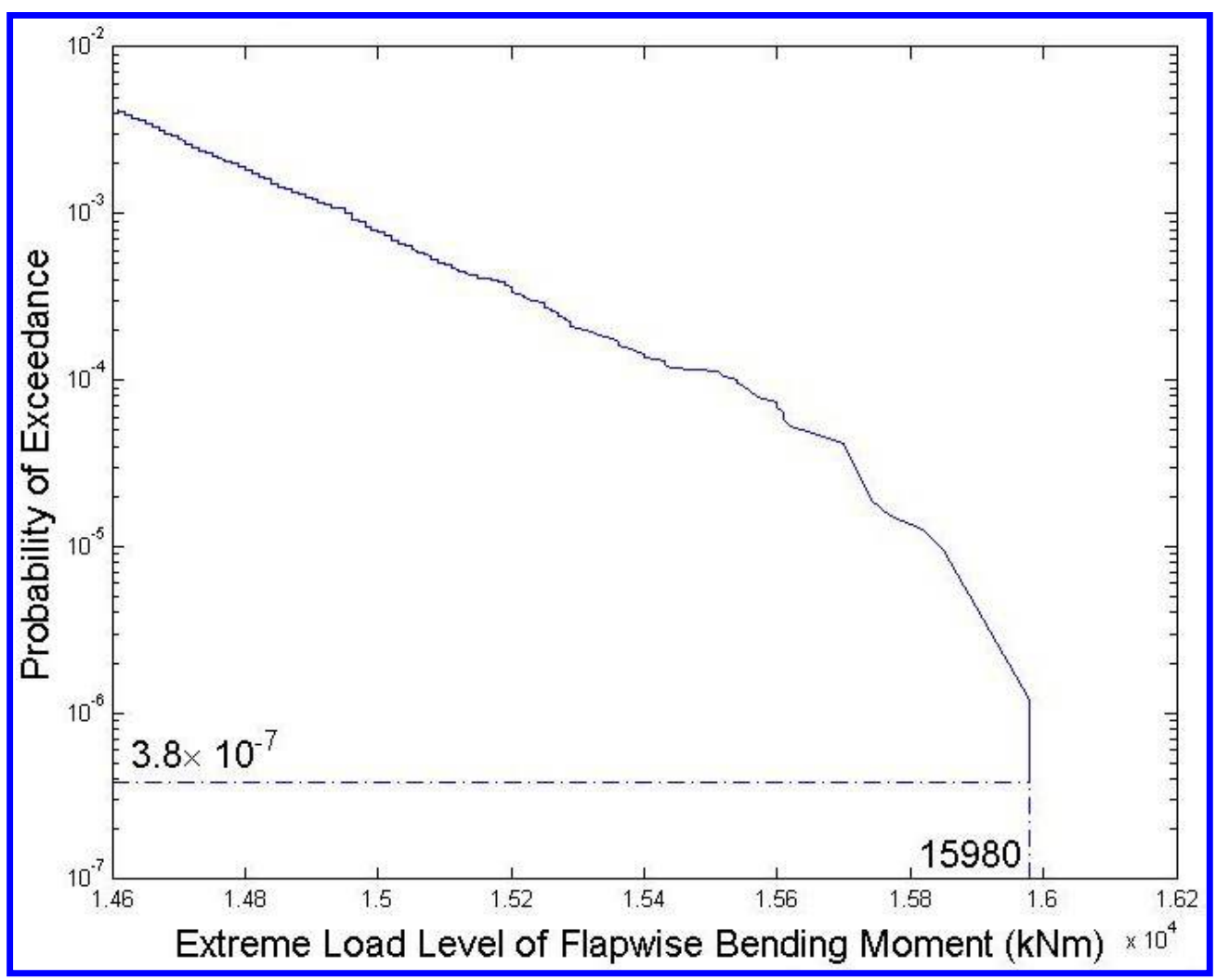

Figure 8: Flapwise bending moment extreme load estimation trajectory 


\section{Conclusion}

In this study, we develop an adaptive algorithm which updates the importance sampling density according to the information obtained from output samples in each iteration. By iteratively increasing parameter $\theta$ in the importance sampling density toward the target extreme quantile $l_{T}$, we improve the simulation efficiency and reduce the estimation uncertainty compared with the existing method.

Our results using the set of NREL simulators demonstrate the superiority of adaptive approach in two aspects over the standard IS method: (a) the proposed approach greatly reduces the extreme quantile estimation variance with the same computational effort; (b) it is capable of estimating much larger extreme load levels with a smaller computational resource. Future studies extend the proposed approach to the extreme load estimation considering wake effects in a multi-turbine wind farm. ${ }^{11}$

\section{References}

${ }^{1}$ Byon, E., Choe, Y., and Yampikulsakul, N., "Adaptive Learning in Time-Variant Processes With Application to Wind Power Systems," IEEE Transactions on Automation Science and Engineering, Vol. 13, No. 2, 2016, pp. 997-1007.

-2Jonkman, B., TurbSim Users Guide: Version 1.50, No. Technical Report NREL/TP-500-46198, National Renewable Energy Laboratory, Golden, Colorado, 2009.

${ }^{3}$ Jonkman, B. and Buhl, M., FAST user's Guide, No. Technical Report NREL/TP-500-38230, National Renewable Energy Laboratory, Golden, Colorado, 2005.

${ }^{4}$ International Electrotechnical Commission, No. IEC/TC88,61400-1 ed.3, Wind Turbines- Part 1: Design Requirements, 2005.

${ }^{5}$ Lee, G., Byon, E., Ntaimo, L., and Ding, Y., "Bayesian spline method for assessing extreme loads on wind turbines," The Annals of Applied Statistics, Vol. 7, No. 4, 2013, pp. 20342061.

${ }^{6}$ Choe, Y., Pan, Q., and Byon, E., "Computationally Efficient Uncertainty Minimization in Wind Turbine Extreme Load Assessments," ASME Journal of Solar Energy Engineering: Including Wind Energy and Building Energy Conservation,, Vol. 138, No. 4, 2016, pp. 0410121.

${ }^{7}$ Manuel, L., Nguyen, H. H., and Barone, M. F., "On the Use of a Large Database of Simulated Wind Turbine Loads to Aid in Assessing Design Standard Provisions," Tech. rep., American Institute of Aeronautics and Astronautics, Grapevine (Dallas/Ft. Worth Region), Texas, 2013.

${ }^{8}$ Cannamela, C., Garnier, J., and Iooss, B., "Controlled stratification for quantile estimation," Annals of Applied Statistics, Vol. 2, No. 4, 2008, pp. 1554-1580.

${ }^{9}$ Choe, Y., Byon, E., and Chen, N., "Importance Sampling for Reliability Evaluation With Stochastic Simulation Models," Technometrics, Vol. 57, No. 3, 2015, pp. 351-361.

${ }^{10}$ Morio, J., "Extreme quantile estimation with nonparametric adaptive importance sampling," Simulation Modelling Practice and Theory, Vol. 27, No. DOI:10.1016/j.simpat.2012.05.008, 2012, pp. 76-89.

${ }^{11}$ You, M., Byon, E., Jin, J., and Lee, G., "When wind travels through turbines: A new statistical approach for characterizing heterogeneous wake effects in multi-turbine wind farms," IISE Transactions, Vol. 49, No. 1, 2017, pp. 84-95. 